\title{
El aprendizaje del álgebra y sus dificultades. Una exploración a través del estudio de errores
}

DANIEL EUDAVE MUÑOZ

Departamento de Educacion/UAA

\section{INTRODUCCION}

Se presentan los fundamentos teóricos y algunos de los resultados de un estudio sobre la frecuencia y naturaleza de los errores algebraicos cometidos por estudiantes de $3^{\circ}$ de secundaria de escuelas públicas de la ciudad de Aguascalientes ${ }^{1}$. El trabajo consistió en la aplicación de una prueba de álgebra a 235 alumnos, la cual sirvió para analizar el tipo de errores cometidos por los alumnos y su frecuencia. En una segunda etapa se desarrollaron entrevistas clínicas a nueve alumnos para explorar la naturaleza de los errores algebraicos. El estudio de los errores nos permite fundamentalmente detectar los temas que representan una mayor dificultad a los alumnos en su aprendizaje y no es, por tanto, una estrategia enfocada a medir la eficiencia de la escuela.

Compartimos la idea de Marilyn Matz de que "los errores son intentos razonables pero no exitosos de adaptar un conocimiento adquirido a una nueva situación". Así, suponemos que el conoci-

1. Daniel Eudave Muñoz, Errores de notación algebraica. Su frecuencia y naturaleza, tesis de Maestría en Educación, Universidad Autónoma de Aguascalientes, México, 1997.

2. M. Matz, "Towards a computational theory of algebraic competence", en The Journal of Mathematical Behavior, no. 3, 1980. 
miento matemático de los alumnos es algo dinámico pero que puede mostrar carencias y en ocasiones operar en contextos inapropiados. Los errores se manifiestan en los alumnos sobre todo cuando se enfrentan a conocimientos novedosos que los obligan a hacer una revisión o reestructuración de lo que ya saben. Hay algunos contenidos que implican reestructuraciones del conocimiento más profundas que otros, así por ejemplo, cuando un niño ya domina la suma de enteros puede presentar errores cuando se le pide hacer operaciones similares a las que realiza sin dificultad, pero con decimales o fracciones. En el nivel de secundaria el paso del aritmética al álgebra es particularmente difícil para la mayoría de los alumnos, y éste es un problema que se presenta prácticamente en todos los escolares del mundo occidental como lo han demostrado diversos estudios. ${ }^{3}$

\section{EDUCACIÓN MATEMÁTICA Y PSICOLOGÍA COGNITIVA}

El soporte teórico de la investigación actual sobre el aprendizaje del álgebra (y de la matemática) se encuentra emparentado con la llamada ciencia cognitiva ${ }^{4}$, que busca dar respuesta a las principales interrogantes en torno a la naturaleza y procesos de la mente (o pensamiento) en todos sus aspectos. Dentro de esta nueva ciencia se acogen los aportes de la psicología, la lingüística, la inteligencia artificial, la antropología, la neurociencia y la filosofía. De estas ciencias, la psicología es la que ha aportado más elementos a los estudios sobre el aprendizaje del álgebra, aunque a últimas fechas los estudios sustentados en la inteligencia artificial y la lingüística son cada vez mayores y hay una tendencia general a buscar puntos explicativos en el contexto cultural de los sujetos, por lo que la an-

3. Ver la reseña que presenta C. Kieran, "The learning and teaching of school algebra", en Crouws, D. (Ed.). Handbook of Research on Mathematics Teaching and Learning, NCTM, MacMillan Publishing Co., New York, 1992.

4. H. Garden, La nueva ciencia de la mente. Paidós, España, 1987. 
tropología ha ocupado en los últimos años un papel importante en el campo de la educación matemática.

En cuanto a los aspectos básicos que la psicología cognitiva contemporánea ha desarrollado para explicar los procesos de aprendizaje de la matemática, citaremos los que señalan De Corte, Greer y Verschaffel: ${ }^{5}$

- La matemática no puede ser considerada como una disciplina singular sino como un conglomerado de subdominios que difieren en sus métodos, tipos de conceptualización y formas de representación;

- La matemática tiene un rol dual, como lenguaje que describe la realidad y como construcción abstracta autónoma;

- La matemática se ha desarrollado (y se desarrolla) como una jerarquía de estructuras en dos planos: el individual y el cultural.

- La matemática depende de sistemas de representación internos (procesos mentales del sujeto) y externos (símbolos, lenguaje natural, representaciones gráficas, objetos físicos, situaciones en las que las personas actúan, etc.).

- En la matemática interactúan dos tipos de conocimientos: conceptual y procedimental.

En consonancia con esta tendencia general del enfoque psicológico del estudio del aprendizaje de la matemática, podemos sintetizar los aspectos más relevantes del álgebra y su aprendizaje:

- El aprendizaje de conceptos: en álgebra, los conceptos básicos son los de variable y ecuación y naturalmente, los conceptos que se retoman de la aritmética como el de número.

- Procedimientos: el álgebra implica el manejo de métodos formales a diferencia del aritmética en donde se aplican algoritmos o incluso se puede recurrir a procedimientos intuitivos.

- Jerarquías y estructuras: los conceptos de variable y ecuación se construyen sobre el concepto de número; para comprender estos tres elementos es necesario que el alumno conozca y maneje en diferentes contextos los principios y reglas que tienen que ver con la operación de estos conceptos, esto es, deben tender redes que les permitan identificar las semejanzas y diferencias

5. Erik de Corte, Brian Greer, Lieven Vershaffel, "Mathematics teaching and learning", en David Berliner, y Robert C. Calfee, (Eds.). Handbook of Educational Psychology, Mcmillan Library Reference, New York, 1996. 
entre ellos. Autores como Kieran ${ }^{6}$ y Resnick, Cauxinille-Marmeche, y Mathieu, ${ }^{\prime}$ afirman que los alumnos deben construir estructuras que les permitan dar un significado a todos los elementos del álgebra (conceptos, representaciones, procedimientos).

- Representaciones: el álgebra es por definición un lenguaje simbólico y formal; sin embargo, sus conceptos y procedimientos pueden expresarse con diferentes tipos de representaciones, como materiales concretos o dibujos, gráficas, figuras geométricas, y esto es particularmente importante para lograr un tránsito adecuado de la aritmética al álgebra. Una gran cantidad de estudios muestra lo difícil que es para los alumnos aprender el álgebra a partir de sus formalizaciones.

\section{EL APRENDIZAJE DEL ÁLGEBRA}

El desarrollo histórico del álgebra ${ }^{8}$ sugiere que la forma más convencional de concebirla es como la rama de las matemáticas que trata con la simbolización de relaciones numéricas generales y estructuras matemáticas y con la operación de esas estructuras. En sentido estricto, toda el álgebra escolar es una "aritmética generalizada" y como tal involucra la formulación y manipulación de una explicación general sobre los números. En sentido amplio, el álgebra puede considerarse como el conocimiento de relaciones y propiedades y como pensamiento matemático en términos generales

6. C. Kieran, "The early learning of algebra: A structural perspective", en Wagner y Kieran (Eds.). Research Issues in the Learning and Teaching of Algebra, NCTM y Lawrence Erlbaum: Reston, VA and Hillsdale, NJ, 1989.

7. L. Resnick, Cauxinille-Marmeche, J. Mathieu, "Understanding algebra", en Thon Sloboda, Rogers Don (Eds.). Cognitive Processes in Mathematics, Oxford Science Publications, 1987.

8. La evolución histórica del álgebra se puede resumir en tres etapas: a) Etapa retórica: antes de Diofanto (s. 250 d.C.), se caracterizó por el uso del lenguaje ordinario para describir la resolución de tipos de problemas particulares y carecer del uso de símbolos especiales para representar incógnitas. b) Álgebra sincopada: iniciada por Diofanto, introduce el uso de letras como cantidades no conocidas (incógnitas). c) Álgebra simbólica: inicia con F. Vieta (1540-1603), en esta etapa es posible expresar soluciones generales y usar el álgebra como una herramienta para proporcionar las reglas que gobiernan las relaciones numéricas. 
más que en términos específicos, sin los requerimientos que acompañan la expresión formal. ${ }^{9}$

El poder y utilidad del álgebra radica en su formulación simbólica, que le permite ser un lenguaje que se autoexplica (que expresa y demuestra sus teoremas). Esta propiedad de constituirse en un sistema de signos "autocontenido" ha hecho que el álgebra sea el lenguaje matemático por antonomasia. ${ }^{10}$

La importancia de la matemática y del álgebra con respecto a otros dominios del conocimiento humano, como señalan Resnick, Cauzinille-Marmeche y Mathieu, ${ }^{11}$ radica en que:

1) La matemática se ocupa de formas de conocimiento abstracto más que cualquier otro campo.

2) El conocimiento matemático está integrado como un lenguaje formal especializado que constriñe el razonamiento matemático y le confiere un extraordinario poder.

3) El lenguaje formal de la matemática juega un doble rol de significante y significado, como el instrumento y como el objeto del razonamiento. En matemáticas, los términos y expresiones de la notación formal tienen una función referencial y formal. Como símbolos referenciales denotan objetos o entidades cognitivas. Como símbolos formales son elementos en un sistema que obedece a reglas propias y no puede funcionar sin la referencia continua a los objetos matemáticos que nombra.

El rol dual de los símbolos matemáticos es particularmente obvio y complicado en el caso del álgebra. El gran poder del álgebra consiste en la posibilidad de realizar una manipulación extensiva de relaciones entre variables dentro de un sistema completamente confiable que no requiere una atención continua al significado referencial de las expresiones intermedias generadas. El hecho de

9. C. Kieran, Op.cit.

10. Teresa Rojano, "El álgebra en el currículum de la secundaria. La reforma de los 90's", en Educación Matemática, vol. 3, no. 3, dic., 1991.

11. L. Resnick et. al., op.cit.

C A A 
que el sistema algebraico pueda "correr en sus propios caminos" es seguramente un factor en favor de su eficiencia. Pero a su vez, las aplicaciones del álgebra en entornos muy concretos le confiere un significado más plausible (y hasta cierto punto, esos entornos o situaciones empíricos son el origen histórico y genético del álgebra).

- El álgebra puede tomar su significado de tres fuentes diferentes:

1) En un nivel, el significado del álgebra está contenido completamente en el sistema formal. Las expresiones que tienen un significado son todas aquellas que éstán bien formadas conforme las condiciones del sistema formal. En este nivel, las expresiones son significativas si pueden derivarse de un conjunto de axiomas y postulados.

2) El álgebra toma en parte su significado del mundo de los números y sus operaciones. Las expresiones algebraicas pueden ser vistas como enunciados de relaciones que pueden ser válidas entre números y operaciones en general. Podemos demostrar que una ecuación algebraica es verdadera o una transformación permitida sustituyendo con números las incógnitas y realizando las operaciones necesarias, aunque por supuesto, de esta forma no son posibles todas las demostraciones.

3) Las expresiones algebraicas y las reglas de transformación también pueden referirse a situaciones reales en que las relaciones entre cantidades juegan un rol; situaciones que pueden matematizarse expresando sus relaciones cuantitativas con una formalización matemática adecuada. Esto es lo que se hace cuando al resolver un problema primero escribimos una ecuación apropiada. También es posible usar una situación real para explicar y justificar las reglas de transformación.

Los diferentes significados de las expresiones y reglas algebraicas hacen surgir una paradoja que es medular en el aprendizaje: por una parte, las expresiones formales toman su significado de las situaciones a que hacen referencia, y por otra, el álgebra obtiene su poder al divorciarse de esas situaciones. Cuando el álgebra es 
usada para resolver problemas, quien resuelve el problema debe primero representarse mentalmente la situación -las cantidades, relaciones y transformaciones involucradas-, y luego escribir la ecuación que la represente usando la notación algebraica. Las transformaciones usadas al resolver las ecuaciones son posibles en parte porque se pone atención a las situaciones específicas que generaron la ecuación, pero acto seguido, sólo se piensa en las operaciones abstractas y las relaciones representadas en la expresión formal. Si las situaciones no son puestas de lado al menos temporalmente, las transformaciones no tendrán sentido.

Las fuentes de significado del álgebra son esquematizadas por Resnick, Cauxinille-Marmeche y Mathieu ${ }^{12}$ de la siguiente forma:

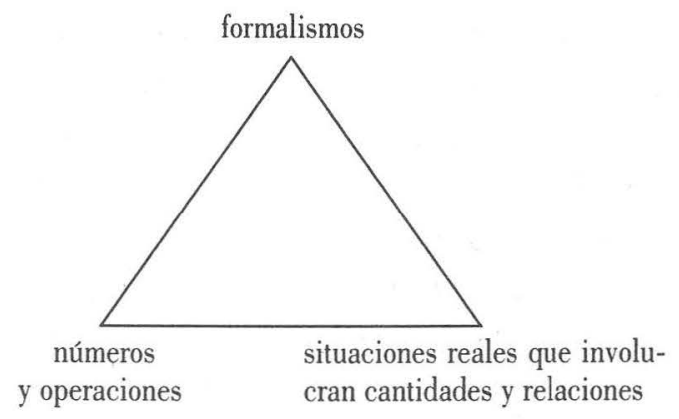

Así pues, tenemos que no es suficiente para un aprendiz del álgebra, querer obtener el significado de las expresiones algebraicas de una sola fuente, sino que hay que complementarlo con las tres para asegurar una comprensión adecuada y flexible.

Complementando lo anterior, estas autoras consideran que son cuatro los principios básicos que sustentan las expresiones y transformaciones del álgebra elemental (entendida como aritmética generalizada), y que deben ser comprendidas y manejadas por los alumnos.

12. Ibidem. 
1) Irrelevancia del orden en la adición. Este principio expresa el hecho de que es permisible sumar cantidades en cualquier orden, sin tomar en cuenta los subgrupos que puedan formarse (como por ejemplo, si se introducen paréntesis) o su orden de presentación. Este principio es presentado en los textos de matemáticas como dos reglas: conmutatividad y asociatividad.

2) Relevancia del orden en la substracción. Este principio es presentado en los libros de texto como la no conmutatividad de la resta.

3) Composición de cantidades dentro de paréntesis. Este principio expresa el hecho de que dos términos adentro del paréntesis son una cantidad singular completa y que esta cantidad puede sumarse $o$ restarse de acuerdo al signo que precede al paréntesis. 0 bien, si el paréntesis aparece al inicio de la expresión, la cantidad compuesta puede servir como el conjunto de inicio del cual el tercer término en la expresión será sumado o restado. Una cantidad compuesta puede además ser comparada o combinada con otra cantidad. Este principio puede usarse para explicar las reglas de cambio de signo al quitar los paréntesis.

4) Composición de transformaciones. Este principio expresa la idea de que transformaciones sucesivas sobre una cantidad dada pueden componer un total singular. Por ejemplo, si $b$ y $c$ se restan de $a$, tal como se indica en la expresión $a-b$ - $c$, es posible componer la transformación en una cantidad singular al substraer $(b+c)$. Este principio ofrece otra forma de justificar la regla de cambio de signos.

Dentro de la matemática hay diferentes tipos de representaciones que favorecen una comprensión más amplia y dinámica de los conceptos, y así por ejemplo, una ecuación de primer grado puede representarse mediante una expresión algebraica o mediante una gráfica, y ambas representaciones son correctas, pero una puede ser más clara para un alumno que otra. Es necesario que para que el alumno tenga una comprensión clara y rica de la matemática, se apoye en los tres vértices del triángulo mostrado arriba, pero final- 
mente debe lograrse una síntesis que debe expresarse en el dominio del formalismo algebraico, pues es una condición para poder acceder a contenidos matemáticos más elaborados. El dominio del lenguaje formal es pues una meta más que un camino, por lo menos en los niveles elementales del aprendizaje de la matemática.

Lograda una comprensión y dominio mínimo de la notación algebraica, esta misma deviene en un campo de significación, pero antes no. Esto lo explica con más detalle James Hiebert ${ }^{13}$ con una teoría sobre el desarrollo de competencias con símbolos matemáticos escritos. Este autor entiende por símbolo "una entidad que se usa para ocupar el lugar de otra entidad. Estas entidades pueden tomar una variedad de formas, desde objetos concretos hasta marcas escritas en papel"14. Los símbolos concernientes a esta teoría son las marcas escritas convencionales para indicar cantidades y operaciones con cantidades. Hiebert señala que es importante hacer notar que los sistemas de símbolos escritos son sólo uno de los lenguajes de la matemática; el lenguaje natural y los modelos concretos como los bloques de Dienes y las regletas de Cuisenaire, son ejemplos de otros sistemas de representación que pueden usarse para describir ideas matemáticas. Aunque hay que hacer notar que en gran medida las matemáticas, sobre todo las escolares, dependen de símbolos escritos.

La teoría de Hiebert propone una sucesión de procesos cognitivos que se acumulan para producir la competencia en el manejo de los símbolos matemáticos escritos. Identifica cinco tipos de procesos básicos; cada tipo de procesos debe emplearse en secuencia. El manejo de los primeros procesos permite la fundación del dominio de los procesos posteriores. Desde este punto de vista los primeros conocimientos y experiencias son cruciales para el aprendizaje

13. James Hieber, "A theory of developing competence with written mathematical symbols", en Educational Studies in Mathematics, vol. 19, no. 3, 1988, pp. 333-335. 14. Ibid., p. 334 . 
posterior. Por lo tanto, la teoría sugiere que muchas de las deficiencias que muestran los estudiantes al trabajar con símbolos escritos se deben a que éstos se involucran en los procesos más avanzados sin tener los fundamentos de los procesos más elementales. El sentido acumulativo de esta teoría indica que los primeros procesos no son descartados, sino empleados como nuevos procesos que se adquieren y usan.

Los cinco procesos principales que contempla la teoría de Hiebert son:

1) Conectar o relacionar los símbolos individuales con sus referentes.

2) Desarrollar procedimientos de manipulación de símbolos.

3) Elaborar procedimientos para los símbolos.

4) Hacer rutinarios los procedimientos con los símbolos.

5) Construir un sistema de símbolos más abstracto.

A continuación se explica cada uno de estos procesos.

1) Conectar o relacionar los símbolos individuales con sus referentes.

En la matemática escolar, los símbolos escritos casi siempre representan cantidades y operaciones con cantidades. Para conectar los símbolos escritos con sus apropiadas referencias cuantitativas los estudiantes deben estar fạmiliarizados con las cantidades más relevantes y las acciones que pueden realizarse con las cantidades, y deben estar familiarizados con los caracteres escritos que deben usar para representar las cantidades y sus operaciones. Y entonces ellos deben crear una correspondencia entre los caracteres escritos y las cantidades o acciones a las que referirán. La familiaridad con las cantidades que habrán de usarse como referentes, es parte del conocimiento informal de muchos niños. Los niños con mucha frecuencia desarrollan actividades con materiales e ideas con las que tienen que identificar cuánto, qué tanto, etc. Estas experiencias co-

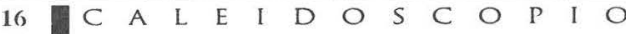


tidianas generan un conocimiento de cantidades y de acciones con cantidades que pueden dar el referente inicial para los símbolos matemáticos escritos. Aún antes de ingresar a la escuela algunos niños adquieren información crucial sobre la escritura de los números, tales como la irrelevancia de los cambios en la apariencia física de un numeral. La competencia con los símbolos escritos inicia con la construcción que los niños hacen de la conexión entre los símbolos individuales y el referente familiar. Los significados de los símbolos individuales son creados como conexiones que se establecen entre las marcas escritas sobre el papel y las cantidades y acciones que se quieren representar. Este proceso implica construir puentes entre símbolos y referentes y cruzar sobre ellos mentalmente muchas veces.

2) Desarrollar procedimientos de manipulación de símbolos.

Los procedimientos son formulados por la manipulación de los referentes de los símbolos individuales observando el resultado y haciendo un paralelo entre la acción con los referentes, con la acción con los símbolos. Por ejemplo, si se representa con los bloques de Dienes a las cantidades 5.1 y 0.36 pueden sumarse mediante un procedimiento natural juntando los bloques del mismo tamaño. Podemos repetir esta acción con símbolos escritos si combinamos los dígitos que están en la misma posición relativa al punto decimal. Entonces, la regla "alinearlos sobre el punto decimal" (que da forma a esta estrategia), tiene su antecedente en la acción de juntar en el mundo de las referencias. De igual forma, otras reglas y algoritmos que se desarrollan como acciones con referentes son ejecutadas, observadas y traducidas al mundo simbólico. En este sentido, los procedimientos simbólicos de las reglas al igual que los símbolos individuales, tienen referentes que les dan su significado.

3) Elaborar procedimientos para los símbolos.

Elaborar procedimientos con símbolos que puedan ser desarrollados y extendidos en nuevas y más complejas tareas, requiere de algunas reflexiones sobre los procedimientos y reglas en sí mismos. 
La elaboración de reglas puede tomar dos diferentes caminos. La elaboración puede hacerse en forma directa si el nuevo problema es equivalente al problema ya conocido en todos los sentidos. Una segunda forma de elaboración involucra una conducta más creativa por parte del estudiante. Es posible que la reflexión sobre la naturaleza de un procedimiento particular estimule el desarrollo de un procedimiento relacionado. Por ejemplo, si el procedimiento que se desarrolla para sumar $36+48$ se basa en el reagrupamiento o recomposición, un procedimiento apropiado puede desarrollarse con el mismo principio para restar 36-19.

4) Hacer rutinarios los procedimientos con los símbolos.

El sistema simbólico se usará más efectivamente si los procedimientos son practicados. Cuando los procedimientos han sido practicados al grado de poder ejecutarlos automáticamente, pueden usarse para lograr una eficiencia máxima. La rutinización de procedimientos necesariamente separa las reglas de sus referentes. La ventaja de la rutinización o automatización de las reglas es que éstas pueden ser ejecutadas con muy poco esfuerzo mental. Los recursos cognitivos pueden reubicarse ante una mayor demanda tanto de un problema como de habilidades de pensamiento de mayor nivel. Dado el limitado espacio disponible para el trabajo cognitivo en los humanos, liberar un espacio adicional es un importante beneficio.

5) Construir un sistema de símbolos más abstracto.

Los sistemas de símbolos pueden desarrollarse en sí mismos por la construcción sobre de otros sistemas. La competencia de los estudiantes con los símbolos continúa su desarrollo como un sistema más abstracto y los caminos en que son construidos sobre las bases de los primeros sistemas son bien reconocidos. Un camino es construir un sistema a través del primigenio, transfiriendo los significados del primero sobre los del sistema posterior. Otro camino se da a través del reconocimiento de la correspondencia entre dos sistemas de símbolos diferentes. Alcanzado este último nivel, el proceso 
de construcción inicia nuevamente, sólo que en un nivel de abstracción mayor. El sistema de símbolos que ya resulta familiar puede ser usado como el soporte para la adquisición de otros significados, en forma similar a la que se utilizó en un primer momento recurriendo a los referentes concretos. El incremento de la competencia requiere el desarrollo y elaboración de procedimientos con símbolos más abstractos. Así pues, la aritmética y la geometría tienen un referente empírico en objetos concretos y cotidianos y esto lo podemos apreciar filogenética y ontogenéticamente. El álgebra se desarrolla sobre las bases de estas dos anteriores; y sobre el álgebra, la aritmética y la geometría se apoyan la trigonometría, la geometría analítica y el cálculo, y así hasta las áreas más abstractas de la matemática contemporánea.

Este proceso que es fácil de explicar, es para la mayoría de los estudiantes muy difícil de transitar. A continuación se analizan las dificultades enfrentadas por los alumnos en el paso de la aritmética al álgebra.

\section{LOS ERRORES ALGEBRAICOS}

Booth ${ }^{15}$ considera que la naturaleza de los errores algebraicos que cometen los niños, es quizás más importante que el tipo de preguntaś que contestan correctamente, especialmente cuando el mismo error es cometido por un gran número de niños. El estudio de los errores es importante porque proporciona información acerca de las formas en que los niños ven el problema y del procedimiento que usan al intentar resolver el problema. Esta información es interesante porque puede sugerir modos de ayuda para que los niños eviten esos errores y también porque puede explicar la aparente falta de progreso en el logro de altos niveles de entendimiento en el álgebra.

15. R. Booth Lesley, Algebra: Children's Strategies and Errors, NFER-NELSON, Londres, 1984. 
El trasfondo de los errores algebraicos (y matemáticos en general) no es de índole exclusivamente pedagógica o didáctica, sino también y sobre todo, epistemológica y psicológica, puesto que "algunos errores son cometidos por un gran número de niños independientemente del tipo de escuela, los libros de texto y los esquemas de enseñanza. Más aún, muchos de esos errores que ocurren regularmente no se restringen a ningún grupo particular de edad y algunos persisten de año en año". ${ }^{16}$

Marilyn Matz, del Centro de Inteligencia Artificial del Instituto Tecnológico de Massachusetts (MIT), desarrolló una teoría para explicar la ocurrencia de dichos errores, resaltando cómo nuevas reglas se construyen desde un conocimiento familiar, o más particularmente, cómo las reglas existentes se generalizan (acertadamente o no) para manejar un amplio rango de información nueva. Para esta autora, la actividad de resolver problemas tiene dos componentes: primero, el conocimiento que precede al nuevo problema usualmente toma la forma de regla que los estudiantes extraen de un prototipo presentado por el maestro o por un libro de texto; segundo, el manejo de técnicas de extrapolación que ayuden a hacer el paso de las reglas conocidas a los problemas poco familiares. "Siendo los errores un reflejo de concepciones incorrectas o ausencia de concepciones en los estudiantes, llama la atención el conocimiento tácito que aplican automáticamente quienes resuelven los problemas". ${ }^{17}$

En el caso de los alumnos que recién inician el estudio del álgebra, el conjunto de conocimientos previos lo integran principalmente la aritmética y la geometría, siendo sobre todo la fijación en los conocimientos y usos aritméticos lo que impide una adecuada comprensión y competencia algebraicas. Pero el problema es aún mayor porque el niño empieza el estudio del álgebra teniendo su pen-

16. K. M. Hart (Ed.). Secondary School Children's Understanding of Mathematics, University of London, Londres, 1980, p. 146.

17. Matz, M., Op.cit., p. 26. 
samiento aritmético no del todo consolidado: "Muchas de las dificultades que los niños presentan en álgebra no son dificultades en el álgebra como tal, pues son más bien dificultades en aritmética" ${ }^{18}$ La falta de formalización del pensamiento aritmético se manifiesta en una comprensión y uso inadecuados de las convenciones de notación aritmética, tales como:

1) El valor de posición (en números enteros y sobre todo en decimales);

2) La notación abreviada mediante exponentes;

3) La escritura de fracciones; $y$

4) La representación de números negativos e irracionales.

Pero es más notoria la falta de comprensión y la falta de formalización en el caso del álgebra, ya que contrariamente a lo que sucede en la aritmética (en donde una buena parte de sus procedimientos se puede realizar sin recurrir a grafismos), en el álgebra es imprescindible el manejo simbólico. Como señala Booth, lo que usualmente se enfatiza más en el aritmética es obtener una respuesta numérica final, y como muchos de los problemas involucran números pequeños, la respuesta final puede obtenerse con procedimientos más intuitivos. En álgebra, por otro lado, -como en los casos más complejos del aritmética, en los que se involucran valores grandes y diferentes a los enteros-, el acercamiento correcto al problema requiere que se precisen aquellos procesos y concepciones que los niños pueden manejar exitosamente al tratar con problemas aritméticos simples. Al toparse con el álgebra, estos niños son confrontados con sus propias carencias de comprensión de los procesos aritméticos. De la misma manera, las confusiones de notación de los niños en álgebra, como todas aquéllas causadas por la omisión de paréntesis, reflejan lo incierto de su comprensión de número. El álgebra hace explícita la concepción incorrecta y la confusión que los niños ya experimentan en aritmética, pero que pueden ser am-

18. Booth, L., Op.cit., p. 87.

$\begin{array}{lllllllllllllll}\text { C } & \text { A } & \text { L } & \text { E } & \text { I } & \text { D } & \text { O } & \text { S } & \text { C } & \text { O } & \text { P } & \text { I } & \text { O } & 21\end{array}$ 
pliamente inadvertidas en ese dominio. Y al mismo tiempo, el álgebra no es aritmética, y no hacer una distinción entre las dos ocasiona en parte las dificultades que los niños enfrentan con ella. Resumiendo, se puede decir que el álgebra, entendida fundamentalmente como aritmética generalizada, puede verse como la escritura de expresiones generales que representan reglas, operaciones y procedimientos aritméticos; no usar o no reconocer estas estructuras en aritmética puede tener considerables efectos sobre el desempeño en el álgebra de los niños.

Por tanto, si el álgebra elemental requiere una revalorización del aritmética, son los aspectos formales del álgebra los que ayudan a desarrollar en el alumno la comprensión completa del aritmética y esto se logra promoviendo antes de la enseñanza del álgebra propiamente dicha, el conocimiento prealgebraico y la extensión de los dominios numéricos de los niños. ${ }^{19} \mathrm{El}$ conocimiento prealgebraico está constituido fundamentalmente por la resolución de ecuaciones de uno o más pasos, en las que basta con operar sobre los datos de la ecuación (sin operar la o las cantidades por determinar). De esta manera se cambia paulatinamente el método aritmético consistente en el análisis de los datos del problema y sus interrelaciones, que se resuelve determinando las operaciones que lo resuelven, por un método prealgebraico que parte del reconocimiento de un problema como un miembro de una familia de problemas y su consecuente modelación con una ecuación, cuyo procedimiento de resolución es independiente de la semántica del enunciado original. Otros tópicos prealgebraicos son el manejo de fórmulas de un cierto nivel de generalidad, el manejo de expresiones numéricas equivalentes, el reconocimiento de patrones numéricos o geométricos, y el registro de situaciones o propiedades generalizadas por el sujeto. La extensión de los dominios numéricos debe abarcar la operatividad con los enteros relativos (con signo) y con los decima-

19. T. Rojano, Op.cit. 
les y las fracciones (racionales), concebidos estos dos últimos también como números con signo, y una cierta explicitación de los aspectos estructurales de estos sistemas numéricos.

El concepto matemático clave de la aritmética es el de número, el cual es enriquecido en el contexto algebraico. Por su parte, los conceptos centrales del álgebra son los de igualdad (que junto con el de número, se retoman de la aritmética), variable, ecuación y función. Esta ampliación conceptual y procedimental se concretiza en el plano simbólico con la permanencia y resignificación de algunas de las convenciones de notación ya existentes y con la introducción de algunas nuevas. Cabe aquí hacer la aclaración de que toda convención de notación como sistema de escritura que es, implica por una parte el uso de símbolos específicos (los referentes de los cunceptos) y por otra, reglas de transformación válidas para cada conjunto de símbolos, esto es, implica una sintaxis que no es de ninguna manera un juego artificioso y arbitrario, puesto que los conceptos mismos en juego, o sea, su semántica, la orientan y condicionan. La distinción entre una sintaxis en apariencia arbitraria y otra con sentido conceptual, la hace Kieran ${ }^{20}$ al considerar como componentes de una expresión algebraica dos tipos de estructuras: por un lado, una estructura superficial, que se refiere a una forma dada de arreglo de los términos y operaciones, sujeta -cuando se arregla secuencialmente- a seguir el orden de las operaciones, y por otro, una estructura sistémica (sistémica en el sentido de relacionarse con el sistema matemático de la cual la expresión hereda sus propiedades), que se refiere a las propiedades de las operaciones, como la conmutatividad y asociatividad, y a la relación entre las operaciones, como la distributividad. La estructura sistémica de las expresiones algebraicas nos permite expresar, por ejemplo, $3(x+2)+5$ equivalente a $5+3(x+2)$ o como $3 x+11$. Así, la estructura de la expresión $3(x+2)+5$ incluye la estructura superficial, que es

20. C. Kieran, Op.cit. 
el ensamble dado de términos y operaciones -en este caso, la multiplicación de 3 por $x+2$, seguida de la adición de 5-, junto con la estructura sistémica, que es, la forma equivalente de la expresión de acuerdo a las propiedades de las operaciones dadas.

A continuación se presentan los principales cambios en las convenciones de notación que se suscitan en el paso del aritmética al álgebra, considerando primeramente los símbolos en juego, para analizar posteriormente los métodos con que los estudiantes tratan de manejar esos símbolos en su intento por comprender y asimilar el pensamiento algebraico.

\section{Los símbolos utilizados}

Prácticamente todos los símbolos que se utilizan en la aritmética se utilizan en el álgebra, pero sufren cambios drásticos en su significado y uso. Entre esos símbolos están:

1. Las letras. En primaria, las letras son utilizadas como abreviaturas para medidas como en el caso de metros (m.), centímetros (cm.); o como iniciales en las fórmulas de áreas y volumen. En álgebra, denotan o bien incógnitas específicas, o bien números generalizados, o bien variables. Los significados que los niños hacen de las letras, tal como lo presenta Küchemann, ${ }^{21}$ reflejan esta transición de un tipo de pensamiento a otro:

Letras como algo que no se usa. En este caso, los niños ignoran las letras, trabajan sólo con los hechos numéricos y eliminan la letra, o bien, reconocen su existencia pero sin darle significado, simplemente la agregan al final de una operación. Incluso los niños pueden inventar reglas para manipular las letras, tales como: sumar todos los números y poner abajo cada letra que ocurre; sumar los núme-

21. Küchemann, "Algebra" en K. M. Hart, Children's Understanding of Mathematics:11-16, John Murray, Londres, 1981. 
ros y poner las letras por cada vez que se presentan en la expresión; sumar todos los números y poner abajo la letra mayor, o la que ocurre más veces.

Letras usadas como objetos. Las letras son vistas como la taquigrafía para un objeto, o como un objeto en sí. Como en el caso de lás mencionadas abreviaturas (m., cm., kg.) o iniciales (a : área; h: altura; $\mathrm{y}:$ yate, yoghurt, etc.).

Letras como un valor específico. A las letras se les asigna un valor numérico desde el comienzo, y por tanto, se cree que cambiar de letra implica cambiar de número. Por ejemplo, algunos niños creen que hay correspondencia entre el orden lineal del alfabeto y el sistema numérico, por lo que creen que las últimas letras del alfabeto tienen un valor más alto que las primeras.

Letras usadas como una incógnita específica. Los niños ven a las letras como un específico pero desconocido número, y pueden operar sobre él directamente. Se presenta, por ejemplo, en operaciones con espacios vacíos, como $3+_{-}=8$, en donde el espacio en blanco sólo puede ser ocupado por un número particular.

Letras usadas como un número generalizado. Se ve a las letras como capaces de tomar varios valores y no uno sólo. No es lo mismo usar $m$ para hacer referencia a "metros" que usar $m$ para representar el número de metros.

Letras usadas como variables. Se ve a las letras como representaciones de un rango de valores no especificados y se ve una relación sistemática entre dos conjuntos semejantes de valores.

2. Signo de "=". La igualdad aritmética, que indica una operación del lado izquierdo y un producto del lado derecho, se transforma en el álgebra en un signo de equivalencia, en donde ambos lados de la expresión vienen a ser lo mismo. Es común que los alumnos que inician el estudio del álgebra lean el signo "=" como una tarea o una pregunta, y el signo de igualdad se presenta como asimétrico. También como lo menciona Matz, en aritmética se le usa como 
tautología y su transformación en igualdad condicionada se da en el contexto de las ecuaciones.

3. Concatenación o yuxtaposición. Esta convención de notación tiene que ver con la posición de los símbolos. En aritmética denota una adición implícita, en el sentido de valor posicional y en la representación de fracciones mixtas (p. ej. $2^{1} \frac{1}{2}=2+1 / 2$ ). En álgebra, esto denota multiplicación: $3 \mathrm{a}=3 \mathrm{x}$ a.

4. Símbolos de "+" y "-.". Estos símbolos en aritmética denotan operación de suma y resta y evocan la ejecución de un algoritmo que da por resultado un tercer número, en álgebra tiene un significado dual: por un lado significan una operación binaria, pero suspendida, y por otro, son interpretados como el signo de un número.

5. Paréntesis. Los paréntesis son indispensables para indicar el orden de ejecución de operaciones en una expresión, sin embargo, los niños ignoran su uso, principalmente porque consideran que es innecesario. Esta creencia se apoya en los siguientes puntos de vista: a) el contexto del problema determina el orden de las operaciones; b) en ausencia de un contexto específico, las operaciones se realizan de izquierda a derecha; c) el mismo valor puede en cada caso obtenerse siguiendo el orden en el cual la secuencia de la operación es realizada (asociatividad de las operaciones).

6. Exponentes. La convención de expresar los exponentes como un pequeño número o letra colocado arriba a la derecha de otro número o letra (llamado base), es interpretado con frecuencia por los niños como multiplicación, esto es: $3^{2}$ lo interpretan como $3 \times 2$, o $3 \wedge n^{22}$ como $3 \times n$. Es interesante que no acepten la yuxtaposición

22. El símbolo “^”"se utiliza para indicar un exponente, así " $\wedge$ " quiere decir que se está elevando la literal "a" a la potencia 1 . 
como multiplicación y en cambio al exponente lo multipliquen por su base, en vez de multiplicar a la base por sí misma el total de veces que lo indique el exponente. Ciertamente, $2^{2}$ es igual a $2 \times 2$, pero es el único caso en que se da esa coincidencia. Posiblemente los niños consideren a los números de tamaño "normal" cuya posición es sobre el renglón de escritura (aunque sea un renglón imaginario), como los números que se "cuentan", mientras que los de tamaño más pequeño, se vean como operaciones especiales aunque no del todo claras, además, es evidente que los niños ven en el exponente un indicador de hacer algo, y no es fácil que acepten al exponente como un elemento de una expresión algebraica, ya que por su formación aritmética, esperan llegar siempre a una respuesta cerrada.

7. Radical. La raíz cuadrada se enseña como una operación antes de que el niño aprenda el álgebra. Al introducirse los radicales en el álgebra, los niños intenta por lo general obtener un resultado numérico sacando la raíz. En ocasiones el símbolo $\sqrt{ }$ es confundido con la división o con la potenciación: al resolver $x+\sqrt{ } 13=\sqrt{ } 13$, un estudiante contestó que la respuesta era 6.5, (dividió 13 entre 2), y cuando se le presentó $\mathrm{x}-\sqrt{3}=0$, contestó que la respuesta era 9 , porque "a 9 le restan 9 , la $\sqrt{3}$ es 9 ".

8. Escritura vertical vs. escritura horizontal. Lo más usual en aritmética es realizar operaciones en forma vertical:

\begin{tabular}{rrr}
6 & 7 & 8 \\
+1 & -2 & $\times 3$ \\
\hline 7 & 5 & $\frac{2}{24}$
\end{tabular}

23. Resultados reportados por Aurora Gallardo y Teresa Rojano, "Áreas de dificultad en la adquisición del lenguaje aritmético-algebraico", en Recherches en Didactique des Mathematiques, vol. 9, no. 2, 1988, pp. 155-188. 
El algoritmo más usual de la división tiene una doble orientación:

125 horizontal para el dividendo, el divisor y el producto

$2 / 250$

05 para los residuos intermedios 0

En algunas ocasiones se usa en el aritmética, una orientación horizontal para realizar las operaciones básicas:

$$
\begin{aligned}
& 2+5=7 \\
& 24-12=12
\end{aligned}
$$

sobre todo se utiliza en operaciones con "espacios vacíos", como

$$
\begin{aligned}
& 2+\square=7 \\
& \square-12=12
\end{aligned}
$$

En álgebra la lectura de expresiones y ecuaciones es principalmente horizontal, aunque la resolución de una ecuación es vertical. En algunos casos se da una relación simétrica, como entre el lado izquierdo y derecho en una ecuación, pero en ocasiones aunque se de la relación de simetría no es apreciada por los niños, por ejemplo, si se pide eliminar los paréntesis de una expresión:

$$
(x+a)(x+b) \Rightarrow x^{2}+a x+x b+a b
$$

o si se pide factorizar:

$$
x^{2}+(a+b) x+a b \Rightarrow(x+a)(x+b)
$$

A estas dos acciones los alumnos las consideran como independientes y, por tanto, transformaciones distintas. 
El álgebra como aritmética generalizada puede verse como la escritura de expresiones generales que representan reglas y operaciones aritméticas conformando un sistema formal con reglas de transformación particulares, por ejemplo:

propiedad asociativa de la suma

$$
(a+b)+c=a+(b+c)
$$

propiedad asociativa de la multiplicación

$$
\text { (ab) } \mathrm{c}=\mathrm{a}(\mathrm{bc})
$$

propiedad conmutativa de la suma

$$
a+b=b+a
$$

propiedad conmutativa de la multiplicación

$$
\mathrm{ab}=\mathrm{ba}
$$

propiedad distributiva de la multiplicación respecto a la suma

$$
\begin{aligned}
& a(b+c)=a b+a c \\
& (b+c) a=b a+c a
\end{aligned}
$$

Los errores algebraicos según Matz, se producen por una sobregeneralización de algunas de las técnicas enseñadas en la escuela que se apoyan en las propiedades anteriores. Sin embargo, los hallazgos de Hart ${ }^{24}$ y Booth ${ }^{25}$ muestran que los niños se resisten a usar las reglas formales que les enseñan en la escuela y prefieren utilizar métodos informales propios, los que funcionan con problemas sencillos pero no se pueden extender a problemas más complejos. Ade-

24. K. M. Hart, Op. cit.

25. L. R. Booth, L.R. Op.cit. 
más, no es fácil simbolizar consistentemente los procedimientos que usan los niños al resolver problemas aritméticos ya que éstos dependen con frecuencia de un contexto determinado. Aún cuando los niños usen un método formal y lo simbolicen correcta y apropiadamente, ellos no pueden apreciar que es apropiado hacerlo.

Los métodos informales que utilizan los niños detectados por Booth son:

1. Intuitivo. No es una reflexión sistemática sobre un conocimiento y por lo tanto no se revisa su consistencia dentro de un conjunto general de conocimiento.

2. Primitivo. Está vinculado estrechamente a las experiencias tempranas en matemáticas. Basado ampliamente en las operaciones de contar, sumar y combinar. Se trabaja únicamente dentro del sistema de números enteros.

3. Ligado al contexto. Se sacan los datos de un problema particular.

4. Indicativos de un manejo elemental y pobre de un método de simbolizar.

Hasta este momento se han analizado las características del proceso de aprendizaje del álgebra y los errores que aparecen como reflejo de las dificultades que hay que superar para lograr un dominio mínimo del álgebra. Veamos ahora desde esta perspectiva los errores de los sujetos de nuestro estudio.

\section{LOS ERRORES MÁS FRECUENTES EN ESTUDIANTES DE SECUNDARIA}

Se utilizó la prueba de álgebra ${ }^{26}$ diseñada y utilizada por el equipo de investigación de Katleen Hart ${ }^{27}$ en una de las investigaciones pioneras sobre los errores algebraicos, la cual está compuesta por 21 preguntas las que a su vez incluyen de uno a cuatro reactivos.

26. Los contenidos de la prueba corresponden en sentido estricto a la preálgebra o aritmética generalizada.

27. K. M. Hart, Op. cit.

30) $\begin{array}{llllllllllllll} & \text { C } & \text { A } & \text { L } & \text { E } & \text { I } & \text { D } & \text { O } & \text { S } & \text { C } & \text { O } & \text { P } & \text { I } & \text { O }\end{array}$ 
Todas las preguntas de la prueba son abiertas y presentan ejemplos de apoyo. El nivel de dificultad de cada reactivo es diferente, contando con cuatro niveles:

Nivel 1: Los reactivos son meramente numéricos o tienen una estructura simple y pueden resolverse usando las letras como objetos o dando un valor específico a la letra, o no usando la letra.

Nivel 2: La diferencia entre estos reactivos y los del nivel anterior es su incremento de complejidad. Se puede decir que el avance hecho en este nivel se debe simplemente al incremento de la familiaridad con la notación algebraica.

Nivel 3: La resolución de los reactivos de este nivel requiere que los alumnos reconozcan los principios de la asociatividad, conmutatividad y distributividad, y que por lo menos reconozcan a las letras como incógnitas específicas.

Nivel 4: En este nivel es necesario que además de cubrir los aspectos mencionados para el nivel 3 , se reconozca y use las letras como variables.

En ésta y la siguiente sección se muestran los resultados correspondientes sólo a una sección de la prueba, que transcribimos a continuación:

$\underline{a+3 a}$ se puede simplificar como $\underline{4 a}$

Simplifica lo más que puedas las siguientes expresiones:

1.- $2 a+5 a=$

2.- $2 a+5 b=$

3.- $(a+b)+a=$

4. $-2 a+5 b+a=$

5.- $(a-b)+b=$

6.- $3 a-(b+a)=$

7.- $a+4+a-4=$

8.- $3 a-b+a=$

9. $-(a+b)+(a-b)=$ 
Para hacer estas simplificaciones es necesario poseer un manejo numérico elemental y conocer las convenciones de notación básicas del álgebra; para los reactivos con nivel de dificultad 3 y 4 se requiere además que los alumnos reconozcan los principios de la adición (asociatividad y conmutatividad).

Se detectaron prácticamente los mismos errores reportados por los autores citados en los primeros apartados. De manera general se aprecia en los alumnos una tendencia a trabajar dentro del conjunto de los números enteros y a usar el conteo y la adición como estrategias básicas, siguiendo procedimientos no formalizados y presentando expresiones sin ningún rigor o apego a las convenciones de notación. La mayoría de los errores encontrados son del tipo que Matz denomina de cambio conceptual y que tienen que ver con el manejo de valores simbólicos, el uso de la notación y la extensión de la interpretación del signo de igual.

Los errores de notación mas sobresalientes son los debidos a un uso inadecuado de los paréntesis, la concatenación, los signos de "+ " y " "", los exponentes y la igualdad. En los reactivos presentados y que solicitan la simplificación de las expresiones, se ven ejemplos de todos estos errores, sobre todo en cuanto al uso inadecuado de exponentes y en el uso de reglas propias. En el reactivo 3 la expresión a simplificar era $(a+b)+a$ y algunos alumnos dieron por respuesta $a^{2} b \circ a^{2}+b \circ$ incluso $a^{2} b^{2}$; en el reactivo 6 , la expresión a simplificar era $3 a-(b+a)$, y se presentaron errores tales como: $3 a^{2} b,-2 a b$, en la primer respuesta se aprecia que los paréntesis son ignorados, mientras que en la segunda, el problema parece radicar en el manejo y comprensión de los signos " + y -".

En cuanto al manejo de las letras, se aprecia una tendencia a usarlas como cosas que se agregan o se ignoran, o en todo caso se les asigna un número (por lo general el 1).

Se presentaron otros errores que aunque no se reportan en la literatura consultada, se pueden considerar como variantes de los errores ya mencionados, y además se presentaron sólo en muy 
pocos alumnos; estos errores son el de la generalización errónea de la regla del binomio cuadrado perfecto, y el uso de subíndices para denotar multiplicación entre números y letras, que es una variante del manejo erróneo de la concatenación.

En cuanto a la generalización inadecuada de la regla del binomio, tenemos varios ejemplos, como en el reactivo 9 en el que había que simplificar la expresión $(a+b)+(a-b)$ : un alumno respondió con la siguiente expresión: $a^{2}-a b+b a-b$; y otro respondió con la expresión $a^{2}-a b+b a-b^{2}$. Estos dos alumnos confundieron la suma con la multiplicación, y esto se debe seguramente a una deficiencia en el manejo de los paréntesis y de los signos "+ y -", y por tanto se puede decir que los alumnos tienen una noción de la estructura superficial de la expresión algebraica en cuestión, tal como lo explica Kieran. De cualquier forma hay que resaltar que estos dos alumnos hasta cierto punto cuidan la simetría del binomio que trataron desarrollar.

Llama la atención la gran variedad de errores que de alguna manera hacen suponer un procedimiento de aplicación repetida y de conteo y agrupación doble de los elementos de una expresión. Por ejemplo, las respuestas a los reactivos 4,6 , y 9, en los que se aprecia una estrategia simple de agrupar, sumar y multiplicar, en un afán de agotar todas las posibles operaciones imaginables para cada expresión:

\begin{tabular}{|l|c|}
\hline Expresión a simplificar & Respuestas \\
\hline $4--2 a+5 b+a$ & $\begin{array}{l}8 \mathrm{a}^{2} \mathrm{~b} \\
8 \mathrm{aba}\end{array}$ \\
\hline 6.- $3 a-(b+a)$ & $\begin{array}{c}3 \mathrm{ab}-3 \mathrm{a}^{2} \\
4 \mathrm{a}-3 \mathrm{ba}\end{array}$ \\
\hline 9.- $(a+b)+(a-b)$ & $\begin{array}{c}\mathrm{a}^{2}-\mathrm{ab} \\
\mathrm{ab}^{2}-a b^{2}\end{array}$ \\
\hline
\end{tabular}

28. C. Kieran, Op. cit. 
Otro aspecto importante, es que en la mayoría de las respuestas erróneas se presentan simultáneamente varios tipos de errores, así por ejemplo, es común encontrar problemas en el manejo de los paréntesis y por consiguiente un manejo inadecuado de la concatenación y la aplicación ingeniosa pero errónea de exponentes y subíndices; casi todos los errores cometidos en los nueve reactivos son muestra de ello. Un ejemplo es la simplificación de la expresión $2 a+5 b$ como $7 \mathrm{a}^{\wedge} 1 \mathrm{~b}^{\wedge} 1$ lo que refleja una comprensión pobre del uso de las letras, la confusión del significado de la concatenación y de los exponentes. En el reactivo 5, en que se solicita simplificar la expresión $(a-b)+b$ un alumno respondió con $a-2 b$, cometiendo un error simple al hacer las sumas sin tomar en cuenta el paréntesis ni el valor de las literales. Con las respuestas $a-b^{2}, b-b^{2}, a^{\wedge}-1 b^{\wedge} 0$, se manifiestan también varios errores simultáneos.

Cuando se analizan estos errores tomando en cuenta el nivel de dificultad, es posible identificar con más precisión las problemáticas subyacentes:

Nivel 1

Este nivel fue el que tuvo un porcentaje mayor de aciertos, y en cuanto a los no aciertos, se presentó un porcentaje alto de errores inclasificables que hace suponer en todo caso errores de procedimiento cometidos por descuido o por no tener muy claras las instrucciones. El porcentaje propiamente de errores de notación, aunque menor, refleja el tipo de dificultades y comprensiones defectuosas del álgebra.

\begin{tabular}{|l|r|}
\hline \multicolumn{2}{|c|}{ 1. Simplifica: 2a $+5 \mathrm{a}$} \\
\hline Acierto: $7 \mathrm{a}$ & $70.6 \%$ \\
\hline Usan el conteo y letras como objetos: $7 ; \mathrm{a}+7$ & $4.3 \%$ \\
\hline Aplicación repetida en el conteo de literales (sobreconteo): $8 \mathrm{a}$ & $15.3 \%$ \\
\hline Errores inclasificables & $5.1 \%$ \\
\hline No contestan & $4.7 \%$ \\
\hline
\end{tabular}


El uso de métodos primitivos como el conteo y el usar las letras como objetos o etiquetas de algo, nos indican una comprensión deficiente que no permite una apropiación del álgebra, y los errores de sobreconteo, que son considerables en el reactivo 1 , nos señalan un intento ingenioso pero inadecuado por hacer algo más que una suma con los elementos de la expresión.

Nivel 2

Más de la mitad de las respuestas a los reactivos de este nivel corresponde a un no acierto (58\%), el cual se compone también de un porcentaje considerable de reactivos sin contestar y de errores de procedimiento, pero también afloran los errores de notación presentados anteriormente. Las respuestas al reactivo 4 confirman también la dificultad con el manejo de las literales:

\begin{tabular}{|l|r|}
\hline \multicolumn{2}{|c|}{ 4. Simplifica la expresión: $2 \mathrm{a}+5 \mathrm{~b}+\mathrm{a}$} \\
\hline Acierto: $3 \mathrm{a}+5 \mathrm{~b}$ & $26.4 \%$ \\
\hline Malinteterpretación de la concatenacion $: 3 \mathrm{a} 5 \mathrm{~b}$ & $2.97 \%$ \\
\hline Uso inadecuado de exponentes: $7 \mathrm{a}^{2} \mathrm{~b} ; 8 \mathrm{a} \mathrm{b} ; 3 \mathrm{a}^{2}+5 \mathrm{~b}$ & $11.5 \%$ \\
\hline Ignoran letras: $2+5+1 ; 8 \mathrm{a} ; 7 \mathrm{a} ; 7 \mathrm{~b}$ & $4.3 \%$ \\
\hline $\begin{array}{l}\text { Hacen operación numérica y sólo agregan la letra usando } \\
\text { inadecuadamente la concatenación: 8ab; 7ab+a; } 9 \mathrm{~b}\end{array}$ & $14.5 \%$ \\
\hline $\begin{array}{l}\text { Aplicacion repetida en el conteo de literales } \\
\text { (sobreconteo): 3a+5ba; 8aba }\end{array}$ & $25.1 \%$ \\
\hline Inclasificables & $3.8 \%$ \\
\hline No contestaron & $11.5 \%$ \\
\hline
\end{tabular}

Considerar a las literales como cosas o abreviaturas de algo que sólo se agregan al resultado de una operación numérica, refleja una comprensión pobre del lenguaje simbólico. Por otro lado, los barroquismos producto del sobreconteo nos harían suponer que los alumnos ni siquiera pueden hacer sumas numéricas, sin embargo los resultados obtenidos en el resto de los reactivos del nivel 1 de 
la prueba muestran esa capacidad elemental de sumar; entonces hemos de suponer que los alumnos ven en las expresiones alfanuméricas entes matemáticos de naturaleza muy diferente a los números que manejan en la aritmética, es algo así como si los alumnos creyeran que las expresiones algebraicas se resuelven mediante la aplicación de alguna regla que buscara agotar todas las posibles combinaciones de elementos.

Nivel 3

Para contestar satisfactoriamente todos los reactivos del nivel 3 es necesario, por lo menos, reconocer en las literales incógnitas específicas e identificar los principios de asociatividad, conmutatividad y distributividad. Los bajos porcentajes de aciertos en este nivel muestran que el dominio de los contenidos propiamente algebraicos es pobre. Los errores que se encontraron en este nivel confirman y amplifican las dificultades encontradas en los niveles anteriores.

Los reactivos 2 y 8 presentan similitudes en su estructura y sus respuestas; los dos son expresiones alfanuméricas en las que es necesario practicar alguna adición y se requiere para resolverlas tener una comprensión clara del significado de la concatenación, del signo "-" y aceptar la "falta de cierre". Veamos las respuestas para cada una:

\begin{tabular}{|l|r|}
\hline \multicolumn{2}{|c|}{ 2.- Simplificar : $2 \mathrm{a}+5 \mathrm{~b}$} \\
\hline Acierto: 2a+5b; no se puede simplificar más & $12.3 \%$ \\
\hline Hacen operaciones numéricas e ignoran letra: 7 & $3 \%$ \\
\hline Hacen operación numérica y agregan letras: $7 \mathrm{ab} ; 7+\mathrm{ab}$ & $66.8 \%$ \\
\hline $\begin{array}{l}\text { Aplicación repetida en el conteo de literales } \\
\text { (sobreconteo): 8xb; 8b }\end{array}$ & $4.3 \%$ \\
\hline Inclasificables & $5.5 \%$ \\
\hline No contestaron & $8.1 \%$ \\
\hline
\end{tabular}




\begin{tabular}{|l|r|}
\hline \multicolumn{2}{|c|}{ 8.- Simplificar: $3 \mathrm{a}-\mathrm{b}+\mathrm{a}$} \\
\hline Acierto: $4 \mathrm{a}-\mathrm{b}$ & $26.0 \%$ \\
\hline Hacen operación numérica e ignoran letra: $3-2+1 ; 5$ & $13.2 \%$ \\
\hline Hacen operación numérica y agregan letra: $3 \mathrm{ab} ; 4 \mathrm{ab} ; 4 \mathrm{ab}$ & $0.9 \%$ \\
\hline Uso inadecuado de exponentes: $4 \mathrm{a}^{2}-\mathrm{b} ; 3 \mathrm{a}{ }^{2} \mathrm{~b}$ & $14.0 \%$ \\
\hline Incomprensión del signo "-": -3ab-4b; $\mathrm{a}-3 \mathrm{~b}$ & $23.4 \%$ \\
\hline Inclasificables & $3.4 \%$ \\
\hline No contestaron & $19.1 \%$ \\
\hline
\end{tabular}

Nivel 4

$\mathrm{Al}$ aumentar la dificultad de los reactivos y por ende las exigencias propiamente algebraicas, aumenta la proporción de respuestas erróneas, aunque los tipos de dificultad siguen siendo los mismos que los aparecidos en los niveles anteriores, como puede apreciarse en el siguiente cuadro:

\begin{tabular}{|c|c|}
\hline 5.- Simplificar: $(\mathrm{a}-\mathrm{b})+\mathrm{b}$ & \\
\hline Acierto: a & $11.5 \%$ \\
\hline Agrupación de elementos: -2ab; 2ba; ab & $4.7 \%$ \\
\hline Uso inadecuado de exponentes: $a b^{2} ; b^{2} ;-b^{2} ; a^{\wedge}-1 b^{\wedge} 0$ & $3.8 \%$ \\
\hline $\begin{array}{l}\text { Uso inadecuado de paréntesis, exponentes y } \\
\text { signos de "+" y "-": } a b-b^{2} ; b-b^{2} ; a+b^{2}\end{array}$ & $13.6 \%$ \\
\hline $\begin{array}{l}\text { Uso inadecuado de paréntesis y signos de "+" y "-": } \\
2 \mathrm{~b}-\mathrm{a} ;-2 \mathrm{~b}+\mathrm{a} ; \mathrm{a}-2 \mathrm{~b}\end{array}$ & $43.3 \%$ \\
\hline Inclasificables: $5 \mathrm{~b} ; 3 ; \mathrm{lb} ; \mathrm{ab}=\mathrm{a}=\mathrm{b}$ & $5.5 \%$ \\
\hline No contestaron & $17.4 \%$ \\
\hline
\end{tabular}

Quien comprenda lo que es la propiedad asociativa de la suma no tiene por que cometer errores como los anteriores, a menos que tenga un desconocimiento total del uso de los paréntesis. Pero por 
otra parte, la enseñanza del uso de los paréntesis puede ayudar a su vez a una mayor comprensión del principio de asociatividad.

Veamos ahora con detalle lo que para algunos alumnos implican estos errores.

\section{LA NATURALEZA DE LOS ERRORES}

Las exploraciones realizadas mediante las entrevistas clínicas nos permitieron confirmar la naturaleza de algunos errores, en el sentido de que nos permitió identificar cuál era el punto de apoyo a partir del cual los alumnos daban significado a las expresiones, esto es, si los alumnos recurrían a sus conocimientos numéricos o a las formalizaciones algebraicas (dos de los vértices del triángulo de Resnick, Cauxinille-Marmeche y Mathieu), ${ }^{29} \mathrm{o}$ bien, por el contrario, identificar en cual de estos dos aspectos tenían más lagunas en su conocimiento.

Una parte de la entrevista consistía en mostrar a los alumnos un material manipulable ${ }^{30}$ con el que es posible representar y manipular las expresiones algebraicas de manera concreta. En los términos de la teoría de Hieber antes señalada, ${ }^{31}$ diremos que es más fácil con el material manipulable conectar a los símbolos con sus referentes. Después de explicarles como hacer las representaciones y operaciones, se les solicitaba a los alumnos resolver con el material aquellos reactivos que no habían podido resolver con lápiz y papel. La mayoría de los alumnos que no pudieron resolver algún ejercicio aún después de que se les explicitó de alguna manera el fundamento numérico o la convención de notación en juego en las expresiones, con el apoyo del material manipulable lograron superar la dificul-

29. L. Resnick, Cauxinille-Marmeche, J. Mathieu, Op.cit.

30. Se utilizó un material diseñado como parte de un conjunto de prácticas de apoyo a la enseñanza de la matemática en secundaria: A. Flores Peñafiel, J. Lerma Rico, A. Martínez Cruz, F. Mirabal, Prácticas de Matemáticas para Segundo de Secundaria, Comunicaciones del cimat, Guanajuato, México, 1987.

31. J. Hieber, Op. cit. 
tad. Lo que al parecer les ayudó a superar los obstáculos fue el reconstruir la secuencia de los pasos señalados como indispensables por Hieber para dotar de sentido a las expresiones simbólicas. El material consiste en unas fichas de colores en las que se tiene escrito un número o literal: solo se manejan dos números, el 1 y el -1 representado por fichas pequeñas de color blanco y negro, respectivamente; las literales $X$ y $Y$ se representan mediante fichas un poco mayores a las de los números, con colores diferentes para cada una y sus respectivos valores negativos, representados con fichas del mismo tamaño pero de color diferente.

Mostraremos a manera de ejemplo dos casos; en primer lugar el de una alumna que pudo superar las dificultades al ayudarle a comprender el sentido de las convenciones de notación con base en los principios de asociatividad, conmutatividad y distributividad; el segundo caso corresponde a otra alumna que sólo hasta que enfrentó de manera concreta las expresiones pudo resolverlas.

\section{Alumna 1}

$\mathrm{Al}$ analizar los resultados a la prueba escrita de esta alumna, encontramos que manejó con coherencia los principios y reglas de la aritmética y del álgebra con expresiones sencillas; tuvo dificultades con los problemas que implican un conocimiento más amplio de igualdad y de variable; no reconoció las propiedades básicas de la suma y la multiplicación en expresiones que incluyen números y literales, aunque sí demostró dominio en aquéllas que presentan únicamente literales; su comprensión de las letras parecía equivalente al uso de incógnitas específicas.

Sin embargo, esta alumna enfrentó algunas dificultades con el manejo de expresiones alfanuméricas durante la primera parte de la entrevista, las que en un primer intento no pudo resolver. Esto se presentó con las expresiones alfanuméricas que tenían que simplificarse y que mostramos anteriormente; contestó erróneamente todos estos reactivos, por lo general procedió haciendo sumas con 
los números y agregando las literales a la suma; su dificultad mayor se presentó con el manejo de los paréntesis y la concatenación. Esto fue lo que aconteció:
A1: Alumna 1.
E: Entrevistador.

E: (Muestra una tarjeta con el reactivo 2, donde se solicita simplificar la expresión 2a+5b).

Al: (La observa y escribe: 7ab).

E: -Ajá, ¿y aquí qué fue lo que hiciste?

Al: -Pues sumé estos dos (refiriéndose a los números), y les sume éstas (refiriéndose a las literales), pero al sumarlas, quedan juntas, pero queda primero a y después b.

E: - ¿Puede quedarte $7 \mathrm{ba}$ ?

Al: -Sí, también puede quedar.

E: - ¿Sí? ¿entonces cuál es la diferencia entre estas dos?

(le muestra las tarjetas con las expresiones $2 \mathrm{a}+5 \mathrm{a}$ y $2 \mathrm{a}+5 \mathrm{~b}$ ).

Al: -Umm ...

E: - ¿Ninguna? bueno, nada más la letra.

Al: -Sí.

E: -Entonces, vamos a ver, ¿ésta cómo quedaría?

(le muestra la tarjeta con la expresión a simplificar: $(a+b)+a)$.

Al: (Escribe: $\left.a^{2}+b a\right)$.

E: -¿Qué fue lo que hiciste?

Al: -Pues multipliqué a por a y b por a.

E: ¿Estás segura que te pide una multiplicación?

Al: -jAy Dios!, (mira la tarjeta), umm ..., (escribe y va diciendo: $\left.\mathrm{a}^{2}+\mathrm{b}\right)$.

E: -Ya no estás multiplicando, ¿verdad?, a ver esta otra

(le entrega la tarjeta con la expresión a simplificar: $2 a+5 b+a)$.

Al: (La observa y escribe: 7ab).

E: -A ver, esta otra (le entrega la tarjeta con la expresión a simplificar: $(a-b)+b)$. 
Al: (Escribe después de observar la tarjeta: (a-b)).

$\mathrm{E}:$-¿Aquí cómo le hiciste?

Al: -No sé.

$\mathrm{E}$ : (Sonríe).

Al: -Es que no, ¿no se multiplica b por ...? es que no, no le entiendo.

E: - ¿No has visto (en la escuela) ejercicios de éstos?

Al: -Es que más bien se me dificulta un poco.

Posteriormente se le pidió a la alumna que resolviera reactivos similares a los anteriores, en los que se hacía uso de números y literales, para ver si en otro contexto, era capaz de manejar las literales. Resolvió correctamente todos esos reactivos, lo que nos hizo suponer que sí reconocía a las literales incluso como variables, que tenía además una noción clara de lo que es la igualdad condicionada, por lo que el problema subyacente en el manejo inadecuado de los paréntesis y la concatenación parecía radicar en el no reconocer las propiedades de la suma y la multiplicación. Por lo tanto, se le pidió que identificara las propiedades asociativa y conmutativa de la suma y la multiplicación y la propiedad distributiva de la multiplicación con respecto a la suma; se le mostraron ejemplos de las propiedades para que dijera si eran verdaderos o no:

$$
\begin{aligned}
& (a+b)+c=a+(b+c) \quad \text { asociatividad de la suma } \\
& a+b=b+a \quad \text { conmutatividad de la suma } \\
& (a b) c=a(b c) \quad \text { asociatividad de la multiplicación } \\
& a b=b a \quad \text { conmutatividad de la multiplicación } \\
& a(b+c)=a b+a c \quad \text { distributividad de la multiplicación } \\
& \text { respecto a la suma } \\
& (b+c) a=b a+c a \quad \text { 6 } \\
& a(b-c)=a b-a c \quad 6 \quad 6606 \\
& (b-c) a=b a-c a \quad \text { "6 }
\end{aligned}
$$


Dio respuestas acertadas en todos los casos, aunque con algunas tuvo que hacer una sustitución de las literales con números para verificarlas. Después de este "repaso", se le volvieron a presentar los reactivos $2,3,4$ y 5 y resolvió rápida y correctamente los tres primeros. Con el reactivo 5 se enfrentó con la dificultad de un número negativo, la cual quiso sortear recurriendo a la regla de los signos sin lograr éxito:
Al: Alumna 1.
E: Entrevistador.

E: (Le presenta la tarjeta con el reactivo 5, simplificar: $(a-b)+b)$.

Al: (Escribe: a-2b).

E: - El paréntesis nos está indicando que primero hay que hacer lo de aquí adentro. En este caso como nada más estás sumando todos los miembros, con la propiedad asociativa puedes cambiar el... Al: - la suma...

E: - no, el paréntesis, o bièn, puedes quitarlo. Y si encierras en el paréntesis a las $b$, ¿cómo quedaría?

Al: (escribe: $a+(-b+b)$

$$
a+(-2 b)
$$

E: -¿Por qué?

Al: - Aquí quedaría así porque aquí es una suma y pues, o sea, todo es una suma. E: - Ajá.

Al: - Entonces aquí $a$ se tiene que sumar con las dos $b$ y quedaría así, $-\mathrm{b}+\mathrm{b}$.

E: - Ajá, y si ésta la simplificas, ¿cómo quedaría?

Al: (Escribe: $a+b)$.

E: - En este caso, aquí ¿qué estas sumando?

Al: - Las $\operatorname{dos} b$.

E: - Ajá, ¿aunque sumes una negativa y una positiva? 
Al: - 0 sea, la ley de los signos es: menos por más da menos, o más por menos da menos, si fueran las dos negativas daba negativo, y si fueran las dos positivas, da positivo.

E: -¿Aunque esté sumando y no multiplicando? porque la ley de los signos es...

A1: - para multiplicar.

E: - Ajá.

A1: - Entonces quedaría $a$.

E: - Sí, ¿por qué?

Al: - No sé, es que no le entiendo.

Se le presentaron nuevamente todas las expresiones, pero ahora con el material manipulable, para saber si era consistente en sus respuestas y ver si era posible que superara la dificultad con los números negativos. Comprendió rápido la forma de representar las expresiones con las fichas y resolvió acertadamente las expresiones 1, 2, 3, $4,7,8,9$. También resolvió las expresiones 5 y 6 , pero tuvo dificultades con el manejo del signo "-" que afectaba lo que se encontraba dentro del paréntesis; aunque con el material manipulable se hace más explícita la propiedad asociativa y por tanto se facilita la simplificación de las expresiones aunque incluyan números negativos, su problema parece ser que se resiste a dejar de usar la regla de los signos, como si las expresiones fueran multiplicaciones:

Al: Alumna 1.

E: Entrevistador.

E: (Le entrega una tarjeta con el reactivo 6 con la expresión a simplificar: $3 a-(b+a))$.

Al: (Representa con fichas de esta manera)

$\mathrm{x}$

$\mathrm{x}-\mathrm{y} \quad \mathrm{x}$

$\mathrm{x}$

$\begin{array}{llllllllllllllll}\text { C } & \text { A } & \text { L } & \text { E } & \text { I } & \text { D } & \text { O } & \text { S } & \text { C } & \text { O } & \text { P } & \text { I } & \text { O } & & 43\end{array}$ 
E: - Ésta ¿cómo quedaría?

$\mathrm{Al}$ : (Mueve las fichas y las deja así)

$$
\begin{array}{ll}
\mathrm{x} & \\
\mathrm{x} & -\mathrm{y} \\
\mathrm{x} & \\
\mathrm{x} &
\end{array}
$$

E: 4a-b, nada más que aquí, hay una complicación que puede pasar desapercibida; con los paréntesis estás encerrando una expresión, y lo que pasa es que te está indicando que todo lo que está dentro se lo vas a restar a $3 \mathrm{a}$, por que si no tuviera el paréntesis, nada más le restarías la $b$, pero aquí como te lo esta encerrando en el paréntesis, todo lo que está adentro se lo restas a $3 a$. Al: - ¡Ah!, entonces sería (manipula fichas)

$$
\begin{aligned}
& \mathrm{X} \\
& \mathrm{x} \\
& \mathrm{x}
\end{aligned}
$$

E: -A ver, vamos a poner la expresión (manipula las fichas)

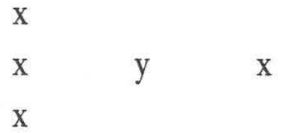

(representa la expresión así)

$\begin{array}{llll}\mathrm{X} & & & \begin{array}{l}\text { sucede que estas dos }(\mathrm{y}, \mathrm{x}) \text { las vas a res- } \\ \text { tar, entonces hay que poner las fichas co- } \\ \mathrm{X}\end{array} \quad-\mathrm{y} \\ \mathrm{x} & & -\mathrm{r} \text { respondientes, con números negativos. }\end{array}$

¿ésta cómo te quedaría?

Al: (Dice: 3a-b-a ).

E: - Ajá, ¿y si simplificas?

A1: (Manipula las fichas y deja la expresión de esta manera)

$\mathbf{X}$

x $\quad-y$

$\mathrm{x}$

E: - Ajá, nada mas eliminaste la x negativa, ¿por fea?

Al: - Es que, joh!, sino también se le puede restar una $x$

44 C 
(representa así la expresión)

$\begin{array}{lll}\mathrm{x} & -\mathrm{y} & -\mathrm{x} \\ \mathrm{x} & & \end{array}$

E: - Pero ¿por qué se la restas a una $\mathrm{x}$ ?

Al: - Porque ya no hay $b$, joh!, se puede quitar ésta: (manipula de nuevo las fichas)

$\mathrm{X}$

X $\quad-\mathrm{X}$

$\mathrm{X}$

E: - No, vamos a ver ahorita con calma.

$\mathrm{X}$

X $\quad \mathrm{y} \quad \mathrm{x}$

$\mathrm{X}$

- te están diciendo: a esto (3x), quiero que le quites esto otro (y, x), o sea, aquí te lo ponen positivo (le señala lo que está dentro del paréntesis), pero aquí con los signos te están diciendo que quieren que lo restes, entonces a $3 \mathrm{a}$ le vas a restar b y a, entonces si le quitaras el paréntesis, esto (lo que está dentro del paréntesis) se representaría con una -b y una -a (lo representa con las fichas)

$\mathrm{X}$

$\mathrm{x}-\mathrm{y} \quad-\mathrm{x}$

$\mathrm{X}$

A1: - Quedaría 3a-b-a

E: - Aja, si le quitas el paréntesis.

Al: - Ajá.

E: - Ahora la puedes simplificar, pern a la $y$ no la puedes simplificar, esa se queda como está.

Al: - Sí.

E: - Pero las x con las x si las puedes agrupar, pero como una es negativa...

Al: - Se eliminan

E: - Se anula una ¿verdad?, entonces tu resultado cuál sería.

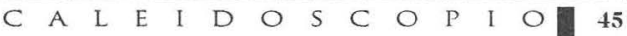


Al: - Umm, 2a-b (representa con las fichas)

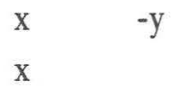

Se pudo comprobar que las dificultades de esta alumna no eran tan grandes como se pensó en un primer momento pues demostró tener una idea muy clara y consistente del sentido ampliado de la igualdad y de la noción de variable. Enfrentó algunas dificultades al no recordar las propiedades asociativa, conmutativa y distributiva, pero luego de hacerle un breve recordatorio de éstas, las pudo aplicar con facilidad y resolver los problemas que requería de su comprensión. El problema que se tardó más en superar fue el manejo del signo "-", pues tuvo dificultad para identificar cuándo era una propiedad o atributo de las expresiones contenidas en un paréntesis y cuándo un operador. Para tratar de superar esto recurrió a la regla de los signos, la cual aplicó de manera descontextualizada, y como si fuera una regla infalible.

\section{Alumna 2}

En general, presentó un dominio de los aspectos aritméticos y geométricos básicos. Por sus respuestas a los reactivos alfanuméricos, nos dio la impresión de que prácticamente desconocía todas las convenciones de notación del álgebra. No reconoció las propiedades asociativa y conmutativa, lo que nos hizo suponer que incluso su comprensión aritmética era limitada. Aparentemente no tenía consolidada la noción de ecuación.

En cuanto a las expresiones a simplificar, pudo contestar las más sencillas, pero en las más complejas procedió haciendo agrupaciones y conteos, o bien, recurriendo a la aplicación fuera de contexto de la regla de los signos:

A2: Alumna 2.

E: Entrevistador 
E: - Resuelve esta otra (entrega tarjeta con reactivo 5 donde le piden simplificar la expresión $(\mathrm{a}-\mathrm{b})+\mathrm{b})$.

A2: (Escribe: $a-2 b)$.

E: - ¿Sumaste?

A2: - Para no poner dos veces la b, sería a-2b.

(...)

E: - ¿Y esta otra? (entrega tarjeta con reactivo 7 para simplificar la expresión $a+4+a-4)$.

A2: - Dos $a$, más cuatro menos cuatro.

E: -A ver, escríbela.

A2: (Escribe: $2 a+4-4)$.

E: - ¿Crees que se pueda simplificar más?

A2: - Sí sería ... (escribe: 4-4+2a ).

E: - No, te estoy pidiendo más bien simplificación.

A2: - Sí, sí, sería ...

(escribe: $-8+2 \mathrm{a})$.

E: - Ajá, pero por qué -8.

A2: - Porque entre los dos cuatros hay un menos, y entre éstos y el dos un más, y más por menos es igual a menos, ¿̨no? ya se me olvidó también eso.

(...)

E: (Da tarjeta con reactivo 8 , simplificar: $3 a-b+a)$.

A2: - Sería cuatro a menos b.

E: - ¿Sería igual que esta otra? (le muestra tarjeta del reactivo 6: simplificar 3a-(b+a)). Esta tampoco la escribiste pero dijiste cuatro $a$ menos $b$.

A2: - Cuatro $a$ menos $b$, es la misma ¿no?, tres $a$ y $a$, igual a cuatro $a$ menos b, es la misma.

E: - ¿Y el paréntesis sale sobrando?

A2: - Sí.

(...)

E: - Bueno, ahora vas a ver otra (le presenta una tarjeta con reactivo 9, simplificar: $(a+b)+(a-b))$. 
A2: - Sería dos $a$ más dos $b$, más por menos igual a más ¿̇no?, dos $a$ más dos $b$.

E: -¿Sí?

A2: - Dos $a$.

A esta alumna le resultaron más entendibles las expresiones a simplificar con el apoyo del material manipulable, de tal suerte que pudo resolverlas todas, aunque algunas le costaron más trabajo:

\section{A2: Alumna 2. \\ E: Entrevistador}

E: - Vamos a ver esta otra (reactivo 7, simplificar a+4+a-4).

A2: (acomoda las fichas: X Y X Y)

E: - Ajá, cómo la simplificarías, a ver, espérate, no, fíjate bien como está la expresión.

A2: (Sólo mueve las mismas fichas dándoles otro orden)

Y X Y X

$\mathrm{E}:-$ ¿Cómo dice?

A2: - y, x, y, x

E: - No, aquí (le señala la tarjeta).

A2: -¡Ah!, aquí, a más cuatro, más a ... jah! es... (cambia una ficha) $\mathrm{X} \quad \mathrm{Y} \quad \mathrm{X}-\mathrm{Y}$

E: - ¿Y cómo quedaría?

$\begin{array}{lll}\text { A2: - Simplificada (mueve las fichas) } & \mathrm{X} & \mathrm{Y}-\mathrm{Y} \\ & \mathrm{X} & \end{array}$

E: - Se puede simplificar más.

A2: (Quita dos fichas, y deja la expresión) X

$\mathrm{X}$

E: - Ajá, dos X, el procedimiento es el correcto, nada más que aquí no es letra, es número, ¿cómo representarías este cuatro? (se refiere a la expresión: a+4+a-4).

A2: - No. 
E: - Lo puedes poner con numeritos.

A2: - No.

E: - ¿Por qué no?

A2: - Con el uno lo puedo representar)

E: - ¿Con el uno? a ver...

A2: (Coloca fichas para representar:

$\mathrm{X}+1 \mathrm{X}+1$

E: - Nada más que fíjate bien como está.

A2: - Es así (cambia una ficha y deja la expresión)

$\mathrm{X}+1 \mathrm{X}-1$

$\mathrm{E}:-$ ¿Cómo quedaría simplificada?

A2: (Quita las fijas que representan +1 y -1 , y deja la expresión) X X

E: - Ajá, de cualquier forma quedan dos X, nada más que sigue pasando un detalle, es: $a$ más cuatro, más, $a$ menos cuatro (con las fichas hace la representación de la expresión)

$$
\begin{array}{llll}
\mathrm{X} & +1+1 & \mathrm{X} & -1-1 \\
& +1+1 & & -1-1
\end{array}
$$

E: - ¿Esto cómo que quedaría?

A2: - Así (quita las fichas de los +1 y los -1): X

$\mathrm{X}$

E: - De cualquier forma te da el mismo resultado.

Aunque el diagnóstico inicial no fue muy favorable y sus respuestas iniciales en la entrevista lo confirmaron, esta alumna pudo superar muchas de las dificultades al quedarle más claro el sentido y función de las convenciones de notación como la concatenación y los paréntesis. Lo que al parecer le ayudó a superar esta situación fue su comprensión de las letras, a las cuales por lo menos en momentos reconocía que podían asumir un valor, lo cual se consolidó al usar el material manipulable, que al parecer le explicitó y/o reforzó esta noción y además le esclareció el mecanismo de las operaciones alfanuméricas. 
Haciendo un balance de los datos presentados, tenemos que en su mayoría:

1) Los. alumnos recurren a estrategias primitivas como la suma con números enteros e incluso a estrategias intuitivas basadas en agrupaciones y conteo de elementos. Son pocos los que utilizan los métodos formales del álgebra.

2) Hay una alta proporción de alumnos que no son capaces de manejar los simbolismos más elementales del álgebra, como el uso de literales, paréntesis, exponentes y los símbolos que experimentan una extensión de su significado como el signo de igualdad, los símbolos "+ y -" y la concatenación, además, casi no se presenta la aceptación de la "falta de cierre".

3) Es particularmente notoria la confusión con la concatenación, pues en principio no se le reconoce como multiplicación y se le usa en diferentes sentidos: para agrupar elementos, para simbolizar un conteo o una suma. La gran cantidad de casos de sobreconteo asociado al manejo inadecuado de la concatenación, hacen suponer que los alumnos intentan hacer algo más que una suma o multiplicación y que recurren a reglas inventadas por ellos o derivadas de algún procedimiento mal comprendido. Podemos afirmar que los alumnos reconocen que el álgebra es algo diferente a la aritmética, pero no saben bien a bien qué es el álgebra, para qué sirve, dónde se aplica, y cómo se realiza.

4) Una gran cantidad de alumnos pretenden resolver los problemas algebraicos agrupando físicamente las literales y los números, como si agruparan objetos físicos; curiosamente no utilizan los paréntesis, siendo que éstos tienen por finalidad agrupar elementos e indicar el orden de las operaciones a realizar. De cualquier forma, a pesar de lo extravagante que puedan parecer los errores, la intuición básica de los alumnos parece aproximarse a la idea algebraica. 
5) La presencia simultánea de varios errores en una sola respuesta parece ser reflejo de una comprensión asistemática del álgebra y de los principios básicos del aritmética.

6) En algunos reactivos se presentó una gran variedad de errores (todos los porcentajes de "inclasificables"), pero en general hay una tendencia a cometer ciertos errores típicos; hay una coincidencia en las reglas y rutinas erróneas. Varios alumnos muestran patrones de error muy sistemáticos, pero en ocasiones los errores parecen respuestas azarosas.

Si vemos estos resultados desde la perspectiva de la teoría de Resnick, Cauxinille-Marmeche y Mathieu ${ }^{32}$ presentada anteriormente, podemos suponer que de las tres fuentes de significados del álgebra señalados por esta autora, es el mundo de los números y sus operaciones el que está dando algún sentido a los intentos de una gran proporción de los alumnos de la muestra. Sin embargo, estos estudiantes no parecen ser muy conscientes de los principios aritméticos básicos implícitos en las operaciones aritméticas y mucho menos en su aplicación a expresiones y transformaciones del álgebra elemental (entendida como aritmética generalizada). Estos principios a los que se hace referencia son los ya mencionados de: a) irrelevancia del orden en la adición; b) relevancia del orden en la substracción; c) composición de cantidades dentro de paréntesis; y d) composición de transformaciones.

Analizando los mismos resultados desde la perspectiva teórica de Hieber, ${ }^{33}$ se puede afirmar tentativamente que la mayoría de los alumnos no han transitado por los cinco procesos que señala este autor, pero con respecto a los contenidos aritméticos; esto quiere decir que aún no consolidan su comprensión aritmética y ya están iniciando el intento de construcción de su comprensión algebraica.

32. Resnick, Cauxinille-Marmeche y Mathieu, Op. cit.

33. J. Hieber, Op.cit. 
Algunos autores como Rojano ${ }^{34}$ y Gallardo y Rojano, ${ }^{35}$ consideran que la preálgebra ayuda a los alumnos a explicitar los principios aritméticos y a consolidar su comprensión del aritmética, pero por los resultados obtenidos, podemos suponer que las lecciones iniciales del álgebra a estos muchachos, no se han orientado de esa forma, sino que al parecer se han centrado en los aspectos formales de la misma. ${ }^{36}$

\section{Conclusiones}

La explicación que aportan Resnick, Cauxinille-Marmeche y Mathieu $^{37}$ sobre las fuentes del significado de las expresiones algebraicas resultó ser muy fructífera en el análisis de nuestros resultados y ofrece interesantes perspectivas para futuros trabajos; las situaciones cotidianas en las que se involucran cantidades y relaciones son tantas y tan variadas, e impregnan el ámbito extraescolar con tal fuerza, que consideramos que deben ser más exploradas. Además hay que tomar en cuenta que en muchas áreas del contexto escolar se hacen aplicaciones de esta ciencia, como por ejemplo en la física, la química, la geografía, el dibujo, por mencionar sólo unas pocas, aunque las interconexiones que existen entre la matemática y todas las demás áreas del conocimiento, por lo general no son explícitamente mostradas a los alumnos.

34. T. Rojano, Op.cit.

35. T. Gallardo y Rojano, Op.cit.

36. El estudio se realizó con alumnos que aún cursaban la secundaria siguiendo los Planes y Programas de la SEP de 1974. En los Planes y Programas vigentes a partir de 1993 se introduce el álgebra en el primer grado de secundaria como preálgebra o aritmética generalizada, con la intención de aminorar los excesos de la formalización con que se venía enseñando el álgebra.

37. Resnick, Cauxinille-Marmeche y Mathieu, Op.cit. 